\title{
1 Variant SARS-CoV-2 mRNA vaccines confer broad neutralization as primary or booster
}

\section{2 series in mice}

3 Authors: Kai Wu, ${ }^{\mathrm{a} *}$ Angela Choi, ${ }^{\text {a }}$ Matthew Koch, ${ }^{\text {a }}$ Sayda Elbashir, ${ }^{\text {ał }}$ LingZhi Ma, ${ }^{\text {a }}$ Diana Lee, ${ }^{\mathrm{a}}$

4 Angela Woods, ${ }^{\text {a }}$ Carole Henry, ${ }^{\mathrm{a}}$ Charis Palandjian, ${ }^{\mathrm{a}}$ Anna Hill, ${ }^{\mathrm{a}}$ Hardik Jani, ${ }^{\mathrm{a}}$ Julian Quinones, ${ }^{\mathrm{a}}$

5 Naveen Nunna, ${ }^{\mathrm{a}}$ Sarah O'Connell, ${ }^{\mathrm{b}}$ Adrian B McDermott, ${ }^{\mathrm{b}}$ Samantha Falcone, ${ }^{\mathrm{a}}$ Elisabeth

6 Narayanan, ${ }^{\mathrm{a}}$ Tonya Colpitts, ${ }^{\mathrm{a}}$ Hamilton Bennett, ${ }^{\mathrm{a}}$ Kizzmekia S Corbett, ${ }^{\mathrm{b}}$ Robert Seder, ${ }^{\mathrm{b}}$ Barney S

7 Graham, ${ }^{\mathrm{b}}$ Guillaume BE Stewart-Jones, ${ }^{\mathrm{a}}$ Andrea Carfi, ${ }^{\mathrm{a}}$ Darin K Edwards ${ }^{\mathrm{a}{ }^{*}}$

9 Affiliations: ${ }^{a}$ Moderna Inc., 200 Technology Square, Cambridge, Massachusetts, 02139, USA;

$10{ }^{b}$ Vaccine Research Center, National Institute of Allergy and Infectious Diseases, National

11 Institutes of Health, 10 Center Dr, Bethesda, Maryland, 20814, USA.

$12{ }^{\ddagger}$ Contributed equally to the manuscript.

$13{ }^{*}$ Corresponding authors: KW (kai.wu@modernatx.com) Moderna Inc. 200 Technology Square

14 Cambridge, Massachusetts, 02139, USA or DKE (darin.edwards@modernatx.com) Moderna Inc.

15200 Technology Square Cambridge, Massachusetts, 02139, USA. 


\section{Abbreviations:}

17 ACE2, angiotensin converting enzyme 2; ELISA, enzyme-linked immunosorbent assay; GMT,

18 geometric mean titer; ID50, inhibitory dilution factor; IgG, immunoglobulin G; LNP, lipid

19 nanoparticle; Nab, neutralizing antibody; NHPs, non-human primates; ns, not significant; NTD,

20 N-terminal domain; PBS, phosphate-buffered saline; PsVN, pseudovirus neutralization titer;

21 RBD, receptor binding domain; RLUs, relative luminescence units; S, spike; SARS-CoV-2,

22 severe acute respiratory syndrome coronavirus 2; TMB, tetramethylbenzidine; UTR, untranslated

23 region; VOC, variant of concern; VOI, variant of interest; VSV, vesicular stomatitis virus. 


\section{Abstract}

25 Severe acute respiratory syndrome coronavirus 2 (SARS-CoV-2) is the causative agent of a global

26 pandemic. Safe and effective COVID-19 vaccines are now available, including mRNA-1273,

27 which has shown 94\% efficacy in prevention of symptomatic COVID-19 disease. However, the emergence of SARS-CoV-2 variants has led to concerns of viral escape from vaccine-induced

29 immunity. Several variants have shown decreased susceptibility to neutralization by vaccineinduced immunity, most notably B.1.351 (Beta), although the overall impact on vaccine efficacy

31 remains to be determined. Here, we present the initial evaluation in mice of 2 updated mRNA

32 vaccines designed to target SARS-CoV-2 variants: (1) monovalent mRNA-1273.351 encodes for and mRNA-1273.351. Both vaccines were evaluated as a 2-dose primary series in mice; mRNA1273.351 was also evaluated as a booster dose in animals previously vaccinated with mRNA-1273.

36 The results demonstrated that a primary vaccination series of mRNA-1273.351 was effective at 37 increasing neutralizing antibody titers against B.1.351, while mRNA-1273.211 was effective at providing broad cross-variant neutralization. A third (booster) dose of mRNA-1273.351

39 significantly increased both wild-type and B.1.351-specific neutralization titers. Both mRNA1273.351 and mRNA-1273.211 are being evaluated in pre-clinical challenge and clinical studies.

41 Keywords: COVID-19, SARS-CoV-2 variants of concern, mRNA-1273, primary series, booster 42 dose, neutralization 


\section{Introduction}

Since the declaration of a global pandemic by the World Health Organization on March 11,

46

47

48

49

50

51

52 2020, infection with the severe acute respiratory syndrome coronavirus 2 (SARS-CoV-2) has led to approximately 4.7 million deaths worldwide $[1,2]$. Shortly after the SARS-CoV-2 genetic sequence was determined, mRNA-1273-a novel lipid nanoparticle (LNP) encapsulated messenger RNA-based vaccine encoding for a prefusion-stabilized, full-length spike (S) glycoprotein of the Wuhan-Hu-1 isolate of SARS-CoV-2-was developed [3, 4]. Vaccination with two $100 \mu \mathrm{g}$ doses of mRNA-1273 four weeks apart was $94 \%$ efficacious against symptomatic COVID-19 disease; mRNA-1273 was granted Emergency Use Authorization by the Food and Drug Administration in December 2020 [5, 6].

The emergence of SARS-CoV-2 variants with substitutions in the receptor binding domain (RBD) and N-terminal domain (NTD) of the viral S protein has raised concerns among scientists and health officials [7-10]. The entry of coronaviruses into host cells is mediated by interaction between the RBD of the viral S protein and the host receptor, angiotensin-converting enzyme 2 (ACE2) [3, 11-14]. Several studies have shown that the RBD is the main target of neutralizing antibodies against SARS-CoV-2 [4, 15-17]. A neutralization "supersite" has also been identified in the NTD [18]. A decrease in vaccine-mediated viral neutralization has been correlated with amino acid substitutions in the RBD (eg, K417T/N, E484K, and N501Y) and NTD (eg, L18F, D80A, D215G, and $\Delta 242-244$ ) of the S protein. Some of the most recently circulating variants of concern (VOCs) and variants of interest (VOIs) with key mutations in the RBD and NTDincluding B.1.1.7 (Alpha), B.1.351 (Beta), P.1 (Gamma), B.1.526 (Iota), and B.1.427/B.1.429 (Epsilon or CAL.20C) lineages-have shown reduced susceptibility to neutralization from convalescent serum and resistance to monoclonal antibodies [18-25]. Note that mutations in the 
67 NTD domain, specifically the neutralization supersite, are extensive in the B.1.351 lineage virus

68

69

70

71

72

73

74

75

76

77

78

79

80

81

82

83

84 [18].

Using 2 orthogonal pseudovirus neutralization (PsVN) assays based on vesicular stomatitis virus (VSV) and lentivirus expressing $\mathrm{S}$ variants, neutralizing capacity of sera from phase 1 participants and non-human primates (NHPs) that received 2 doses of mRNA-1273 was reported [26]. No significant impact on neutralization against the B.1.1.7 variant was observed. However, reduced neutralization was measured against the B.1.351 variant, and to a lesser extent, in the P.1 B.1.427/B.1.429 and B.1.1.7+E484K variants [26]. Clinical studies in South Africa demonstrated reduced efficacy against symptomatic COVID-19 disease for the NVX-CoV2373 (Novavax), AZD1222 (University of Oxford/AstraZeneca), and Ad26.COV2.S (Janssen/Johnson \& Johnson) vaccines [27-30]. Pfizer/BioNTech has recently reported high efficacy of the mRNA BNT162b2 vaccine against B.1.351 among a small number of recipients from South Africa in the phase 2-3 portion of a global phase 1-2-3 trial; however, a report from Israel suggests increased breakthrough infection rates by B.1.351 in BNT162b2 vaccinated individuals at 7-14 days after the second dose $[31,32]$. Studies have demonstrated reduced neutralization titers against the full B.1.351 variant following mRNA-1273 vaccination, although levels are still significant and expected to be protective based on challenge studies in NHPs [26, 33, 34]. Despite this prediction of continued efficacy of mRNA-1273 against this key variant of concern (VOC), the magnitude and duration of vaccine-mediated protection is still unknown. Moreover, a key related question is whether development of new mRNA vaccines to match the B.1.351 variant will enable enhanced neutralization responses and durability.

Herein, we present the design and pre-clinical evaluation of updated mRNA-1273 vaccines against SARS-CoV-2 variants, which include monovalent mRNA-1273.351 and a multivalent 
90 mRNA-1273.211. Like mRNA-1273, mRNA-1273.351 encodes the prefusion stabilized S protein

91 of SARS-CoV-2; however, in contrast to mRNA-1273, mRNA-1273.351 incorporates key

92 mutations present in the B.1.351 variant, including L18F, D80A, D215G, $\Delta 242-244$, R246I,

$93 \mathrm{~K} 417 \mathrm{~N}, \mathrm{E} 484 \mathrm{~K}, \mathrm{~N} 501 \mathrm{Y}, \mathrm{D} 614 \mathrm{G}$, and A701V (Fig. 1). To expand the breadth of coverage to

94 multiple circulating variants as well as the ancestral wild-type virus that is still circulating globally,

95 mRNA-1273.211 is a 1:1 mix of mRNA-1273 and mRNA-1273.351. Two initial studies were

96 performed in BALB/c mice to evaluate the immunogenicity of mRNA-1273.351 or mRNA-

971273.211 as a primary series and/or as a booster. The first study assessed the immunogenicity of

98 a primary series (day 0,21 ) of mRNA-1273.351 or mRNA-1273.211 versus mRNA-1273. A

99 second study evaluated the immunogenicity of a third (booster) dose of mRNA-1273.351213 days

100 after mice were vaccinated with a primary series of mRNA-1273, and immunogenicity was

101 assessed before and after the booster dose. 

$-70^{\circ} \mathrm{C}$ until further use. The drug product underwent analytical characterization-which included

\section{Methods}

\subsection{Data reporting}

No statistical methods were used to predetermine sample size. The experiments were not randomized and the investigators were not blinded to allocation during experiments and outcome assessment.

\subsection{Pre-clinical vaccine $m R N A$ and LNP production process}

A sequence-optimized mRNA encoding prefusion-stabilized Wuhan-Hu-1 or B.1.351-variant SARS-CoV-2 S-2P protein was synthesized in vitro using an optimized T7 RNA polymerasemediated transcription reaction with complete replacement of uridine by N1m-pseudouridine [35]. The reaction included a DNA template containing the immunogen open-reading frame flanked by 5' untranslated region (UTR) and 3' UTR sequences and was terminated by an encoded polyA tail. After transcription, the cap-1 structure was added to the 5' end using the Vaccinia capping enzyme (New England Biolabs) and Vaccinia 2'-O-methyltransferase (New England Biolabs). The mRNA was purified by oligo-dT affinity purification, buffer exchanged by tangential flow filtration into sodium acetate, $\mathrm{pH} 5.0$, sterile filtered, and kept frozen at $-20^{\circ} \mathrm{C}$ until further use.

The mRNA was encapsulated in an LNP through a modified ethanol-drop nanoprecipitation process described previously [36]. Ionizable, structural, helper, and polyethylene glycol lipids were briefly mixed with mRNA in an acetate buffer, $\mathrm{pH}$ 5.0, at a ratio of 2.5:1 (lipids:mRNA). The mixture was neutralized with Tris- $\mathrm{HCl}, \mathrm{pH} 7.5$, sucrose was added as a cryoprotectant, and the final solution was sterile-filtered. Vials were filled with formulated LNP and stored frozen at the determination of particle size and polydispersity, encapsulation, mRNA purity, double- 
124 stranded RNA content, osmolality, $\mathrm{pH}$, endotoxin, and bioburden-and the material was deemed 125 acceptable for in vivo study.

\subsection{Mouse model}

Animal experiments were carried out in compliance with approval from the Animal Care and Use Committee of Moderna Inc. Female BALB/c mice (6 to 8 weeks old; Charles River Laboratories) were used. mRNA formulations were diluted in $50 \mu \mathrm{L}$ of $1 \mathrm{X}$ phosphate-buffered saline (PBS), and mice were inoculated via intramuscular injection into the same hind leg for both prime, boost, and third dose. Control mice received PBS because prior studies have demonstrated that tested mRNA formulations do not create significant levels of non-specific immunity beyond a few days [37-39]. Sample size for animal experiments was determined on the basis of criteria set by the institutional Animal Care and Use Committee. Experiments were neither randomized nor blinded.

\subsection{Enzyme-linked Immunosorbent Assay (ELISA)}

Microtiter plates (96-well; Thermo) were coated with $1 \mu \mathrm{g} / \mathrm{mL}$ S-2P protein (Genscript) corresponding to the $\mathrm{S}$ protein of the Wuhan-Hu-1 virus. After overnight incubation at $4{ }^{\circ} \mathrm{C}$, plates were washed four times with $\mathrm{PBS} / 0.05 \%$ Tween-20 and blocked for 1.5 hours at $37^{\circ} \mathrm{C}$ (SuperBlock-Thermo). After washing, five-fold serial dilutions of mouse serum were added (assay diluent: $0.05 \%$ Tween-20 and 5\% goat serum in PBS). Plates were incubated for 2 hours at $37^{\circ} \mathrm{C}$, washed and horseradish peroxidase-conjugated goat anti-mouse immunoglobulin G (IgG) (Southern Biotech) was added at a 1:20,000 dilution (S-2P) in assay diluent. Plates were incubated for 1 hour at $37^{\circ} \mathrm{C}$, washed, and bound antibody was detected with a 3,3',5,5'-tetramethylbenzidine (TMB) substrate (SeraCare). After incubation for 10 minutes at room temperature, the reaction was stopped by adding a TMB stop solution (SeraCare) and the absorbance was measured at 450 
147 nm. Titers were determined using a four-parameter logistic curve fit in Prism v.8 (GraphPad

148 Software, Inc.) and defined as the reciprocal dilution at approximately optical density $450=1.0$

149 (normalized to a mouse standard on each plate).

150

151

152

153

154

155

156

157

158

159

160

161

162

163

164

165

166

167

168

\subsection{Recombinant VSV-based PsVN assay}

Codon-optimized full-length $\mathrm{S}$ protein of the original Wuhan-Hu-1 variant with D614G mutation (D614G) or the indicated S variants, listed in Table 1, were cloned into a pCAGGS vector. To make SARS-CoV-2 full-length S pseudotyped recombinant VSV- $\Delta$ G-firefly luciferase virus, BHK-21/WI-2 cells (Kerafast) were transfected with the S expression plasmid and subsequently infected with VSV $\Delta$ G-firefly-luciferase as previously described [40]. For a neutralization assay, serially diluted serum samples were mixed with pseudovirus and incubated at $37^{\circ} \mathrm{C}$ for 45 minutes. The virus-serum mix was subsequently used to infect A549-hACE2-TMPRSS2 cells [41] for 18 hours at $37^{\circ} \mathrm{C}$ before adding ONE-Glo reagent (Promega) for measurement of the luciferase signal by relative luminescence units (RLUs). The percentage of neutralization was calculated based on the RLUs of the virus-only control, and subsequently analyzed using four-parameter logistic curve (Prism v.8).

\subsection{Statistical Analysis.}

Animal studies were completed once with all in vitro testing completed in duplicate or triplicate with 1 replicate, unless otherwise stated. Two-sided Wilcoxon matched-pairs signed rank test was used to compare the same animals against different viruses or at different time points. Statistical analyses were performed (Prism v.8). Geometric mean titers with 95\% CIs and lower limits of detection are included, where applicable. 
3. Results series in mice wild-type and variant viruses (Table 1).

first and second injection, with 4.5 to 9.4-fold increased S-2P binding titers measured after the second dose (Fig. 2b). Slightly lower antibody levels were observed for mRNA-1273.351 compared with mRNA-1273, potentially due to the coating S-2P protein used in the ELISA being homologous to mRNA-1273. These results demonstrate that both mRNA-1273.351 and mRNA1273.211 are immunogenic in mice. mRNA-1273 elicited higher neutralization titers against the smaller than previously measured with phase 1 clinical trial sera [26]. mRNA-1273.351 elicited higher neutralization titers against B.1.351 compared with the D614G pseudovirus (Fig. 2c,d), with an approximate 4-fold difference in measured titers. When mRNA-1273.211 was used to 
192 of mice with mRNA-1273.351 elicited high levels of neutralizing antibody against the B.1.351

193 pseudovirus and comparably lower levels versus D614G, whereas the multivalent mRNA-

194 1273.211 vaccine stimulated robust neutralization responses against both D614G and B.1.351

195 pseudoviruses.

196 Sera from mice collected 2 weeks after the second injection was also assessed against SARS-

197 CoV-2 variants that emerged in Brazil (P.1) and California (B.1.427/B.1.429 or CAL.20C). As

198 described in Table 1, some of the mutations in these variants were different from both the Wuhan-

199 Hu-1 and B.1.351 lineages, although the RBD mutations (K417T/N, E484K, N501Y) are common

200 to both the P.1 and B.1.351 viruses. As observed in previous assessments of NHPs and clinical trial

201 sera [26], mice vaccinated with mRNA-1273 showed an approximate 2-fold reduction in

202 neutralizing antibody levels against both the CAL.20C and P.1 variants (Fig. 2e). These reductions

203 in neutralization titers against CAL.20C and P.1 variants were more pronounced in mice vaccinated

204 with mRNA-1273.351 (3.7-fold and 2.6-fold reductions in geometric mean titers [GMTs] for

205 mRNA-1273.351 compared with mRNA-1273, respectively). However, the multivalent mRNA-

2061273.211 vaccine neutralized these variants similarly to mRNA-1273.

207

208

209

210

211

212

213
$3.2 \mathrm{mRNA}-1273.351$ was an effective third (booster) dose in animals previously vaccinated with a primary vaccination series of $m R N A-1273$

To evaluate the ability of the mRNA-1273.351 to boost pre-existing immunity and increase neutralization against both the wild-type and the B.1.351 virus, BALB/c mice were immunized with 1 or $0.1 \mu \mathrm{g}$ mRNA-1273 on day 1 and 22, and the level and durability of the antibody responses were evaluated over the course of 7 months. Sera collection occurred on day 212 , and a third dose of 1 or $0.1 \mu \mathrm{g}$ mRNA-1273.351 was administered on day 213 (Fig. 3a). 
High levels of binding antibody were elicited by vaccination with mRNA-1273, with peak

215 titers measured 2 weeks after the second dose (Fig. 3b). After an initial drop in antibody levels,

216 titers were stable over the 7-month monitoring period. Following the mRNA-1273.351 booster

217 injection, antibody levels dramatically rose, exceeding the previously measured peak for both the

$218 \quad 1$ and $0.1 \mu \mathrm{g}$ dose levels.

219 Neutralization titers were measured in the D614G PsVN assay on day 36 and 212, 1 day prior

220 to the third dose. Titers remained high, with an $\sim 1.5$-fold drop measured over that period. Note

221 that neutralizing titers at day 212 were measured in both the D614G and B.1.351 PsVN assays

222 (Fig. 3c-e), with 6.6-fold higher titers measured in the D614G PsVN assay; this difference is

223 similar to neutralization reductions observed with sera of NHPs and humans who received 2 doses

224 of mRNA-1273 and increases the potential correlation of this animal model to what may be

225 observed in humans.

226 The third dose of mRNA-1273.351 increased neutralization titers 4.5 - and 15-fold in the

227 D614G and B.1.351 PsVN assays, respectively (Fig. 3c,d). The difference between the 2 assays

228 narrowed to 2-fold following the booster dose (Fig. 3c,e); the GMT of 15,524 against B.1.351 was

$229 \sim 1.5$ fold higher than the peak titer against D614G 2 weeks after the second dose (Fig. 3c). Animals

230 vaccinated at the $0.1 \mu \mathrm{g}$ dose level had lower titers, but similar trends for binding antibody and 231 neutralization titers were observed (data not shown). 


\section{Discussion}

In this study, mRNA-1273.351 and mRNA-1273.211 were evaluated in mice as both a primary vaccination series and as a third booster dose in animals previously vaccinated with 2 injections of mRNA-1273. As a primary vaccination series, both vaccines were potently immunogenic after

237 after the second injection. Neutralizing activity of mRNA-1273.351 against the D614G variant was 4-fold lower than that against the B.1.351 variant and 6.3-fold lower against D614G variant compared to mRNA-1273. In contrast, the multivalent mRNA-1273.211 vaccine elicited robust and comparable neutralizing titers against both D614G and B.1.351, which closely match those observed against D614G after mRNA-1273 vaccination. Thus, as a primary vaccination series, a multivalent approach appears most effective in broadening immune responses-as neutralization potency was enhanced against both B.1.351 and P.1 versus mRNA-1273 and remained significant against B.1.427/B.1.429/CAL.20C. approximately 7 months previously. The third dose of mRNA-1273.351 dramatically boosted both

247 S-2P binding antibody titers (Fig. 3a,b) and D614G and B.1.351 PsV neutralization titers (Fig. 3c-

248 e). Neutralizing titers against B.1.351 PsV increased to levels well above the peak neutralizing 249 titer against D614G after the second dose of mRNA-1273, the latter of which was fully protective in mice challenged with the mouse-adapted USA-WA1-F6/2020 variant [41]. In addition, the

251 booster dose also increased neutralizing titers against D614G, although the fold-increase was less

252 than that against B.1.351, as expected. Overall, the third injection of mRNA-1273.351

253 dramatically increased both D614G and B.1.351 neutralization titers, with titers much higher than 254 the day 36 peak. Further, the difference in titers measured in the D614G and B.1.351 PsVN assays 
255 decreased from a 6.6-fold difference prior to the boosting dose, to a 2-fold difference 2 weeks after 256 the third dose.

257 The number of animals available for boosting in this study allowed evaluation of only 1 258 boosting scenario (ie, mRNA-1273.351). Ongoing studies will evaluate the ability of mRNA-1273, 259 mRNA-1273.351, and mRNA-1273.211 to effectively boost immunity driven by a primary 260 vaccination series of mRNA-1273. Studies will also evaluate mRNA-1273.351 and mRNA$261 \quad 1273.211$ in additional primary vaccination and boosting studies in mice, golden Syrian hamsters, 262 and rhesus macaques, with either Wuhan-Hu-1 or B.1.351 challenge planned. These studies are 263 designed to assess the level of neutralization of sera derived from vaccinated animals against 264 pseudoviruses with either the Wuhan-Hu-1 D614G or the B.1.351 S proteins and the level of 265 protection provided against Wuhan-Hu-1 and B.1.351 challenge. Global surveillance for the 266 emergence of additional SARS-CoV-2 VOCs and the neutralization of VOCs by mRNA-1273

267 vaccinee sera are also ongoing. If additional variants emerge that reduce the neutralization capacity 268 of mRNA-1273 further, additional mRNA vaccine designs may be developed and evaluated. In 269 this study, the multivalent mRNA-1273.211 vaccine has already demonstrated to be an 270 immunogenic strategy against multiple variants, and ongoing preclinical and clinical studies will 271 provide further evidence of the utility of using a booster dose of mRNA-1273.211. This approach 272 also supports seasonal adjustment, allowing for changes in response to the evolution of the SARS273 CoV-2 virus.

274 Several potential limitations to the current study should be highlighted. Sera from mRNA-1273 275 vaccinated NHP or human sera was shown to have 6-8 fold reduced neutralizing activity against 276 the B.1.351 variant SARS-CoV-2 in several assessments, although the level of neutralization 277 remained at levels that are predicted to be protective [26]. Results in this study, however, showed 
278 that after the primary series of mRNA-1273, only a 2-fold reduced neutralization against the B.1.351 virus was evident 2 weeks after the second dose. A 6.6-fold reduction was seen at day 212, more relevant to what has been measured from human sera. Further studies in animal species more predictive of responses in humans such as NHPs as well as clinical studies in humans are ongoing. Further, only mRNA-1273.351 was evaluated as a third dose. The ability of the multivalent mRNA-1273.211 vaccine to boost immunity against both the D614G and B.1.351 viruses has not yet been assessed in preclinical models, although a recent exploratory analysis among a subset of patients in a clinical trial showed a mRNA-1273.211 booster dose increased neutralization against dose. This level of durability may not be reflective of that measured in NHPs or humans [42,43]. natural or vaccine-induced immunity served as a call to action. Not only are continued vaccination 291 efforts needed to prevent the emergence of future VOCs, but strategies are needed for new SARS292 CoV-2 vaccine research and development that can enhance the level of protection against key 293 VOCs should they arise. The mRNA vaccine platform approach against SARS-CoV-2 VOCs has 294 now been demonstrated in mice to be effective at broadening neutralization across variants and to 295 boost antibody levels when applied as a third dose, with mitigation of the significant reduction in 296 neutralization seen against the B.1.351 lineage. The mRNA platform allows for rapid design of 297 vaccine antigens that incorporate key mutations, allowing for rapid future development of 298 alternative variant-matched vaccines should they be needed. The designs evaluated in this study 
300 immune response through boosting; additional VOC designs can be rapidly developed and

301 deployed in the future if needed to address the evolving SARS-CoV-2 virus.

\section{Declaration of Competing Interests}

H.B., G.B.E.S.-J., A.C., and D.K.E. are employees of and shareholders in Moderna Inc.

S.O.C., A.B.M., K.S.C., R.S., and B.S.G. report no conflict of interest.

\section{Acknowledgements}

308 We thank Michael Brunner and Dr. Michael Whitt for kind support on recombinant VSV-based

SARS-CoV-2 pseudovirus production. Medical writing and editorial assistance was provided by

311 guidelines, funded by Moderna Inc, and under the direction of the authors.

\section{Contributors}

313 Conceptualization, K.W., S.E., S.R., E.N., G. S.-J., A.C., D.K.E.; data collection, K.W., A.C.,

314 M.K., S.E., L.M., D.L., A.W., C.H., C.P., A.H., H.J., J.Q., N.N., S.O., A.M.; analysis/interpretation

315 of data, K.W., A.C., M.K., A.W., C.H., C.P., D.K.E.; writing—original draft preparation, D.K.E.;

316 reviewing and editing, all authors. All authors have read and agreed to the published version of the

317 manuscript.

\section{Data Availability}

319 The authors declare that the data supporting the findings of this study are available within this article. 


\section{Role of the Funding Source}

322 Employees of the study sponsor, Moderna, Inc., contributed to the study design, data collection,

323 analysis and interpretation, and writing of the report.

\section{$324 \quad$ Funding}

325 This research was funded by Moderna Inc. 


\section{References}

[1] Zhou P, Yang XL, Wang XG, Hu B, Zhang L, Zhang W, et al. A pneumonia outbreak associated with a new coronavirus of probable bat origin. Nature. 2020;579:270-3.

[2] Coronaviridae Study Group. The species Severe acute respiratory syndrome-related coronavirus: classifying 2019-nCoV and naming it SARS-CoV-2. Nat Microbiol. 2020;5:536-44.

[3] Wrapp D, Wang N, Corbett KS, Goldsmith JA, Hsieh CL, Abiona O, et al. Cryo-EM structure of the 2019-nCoV spike in the prefusion conformation. Science. 2020;367:1260-3.

[4] Jackson LA, Anderson EJ, Rouphael NG, Roberts PC, Makhene M, Coler RN, et al. An mRNA Vaccine against SARS-CoV-2 - Preliminary Report. N Engl J Med. 2020;383:1920-31.

[5] Baden LR, El Sahly HM, Essink B, Kotloff K, Frey S, Novak R, et al. Efficacy and Safety of the mRNA-1273 SARS-CoV-2 Vaccine. N Engl J Med. 2021;384:403-16.

[6] FDA (2021) COVID-19 Vaccines. Accessed April 13, 2021.

https://www.fda.gov/media/144673/download.

[7] Korber B, Fischer WM, Gnanakaran S, Yoon H, Theiler J, Abfalterer W, et al. Tracking Changes in SARS-CoV-2 Spike: Evidence that D614G Increases Infectivity of the COVID-19 Virus. Cell. 2020;182:812-27 e19.

[8] Plante JA, Liu Y, Liu J, Xia H, Johnson BA, Lokugamage KG, et al. Spike mutation D614G alters SARS-CoV-2 fitness. Nature. 2021;592:116-21.

[9] Volz E, Hill V, McCrone JT, Price A, Jorgensen D, O'Toole A, et al. Evaluating the Effects of SARS-CoV-2 Spike Mutation D614G on Transmissibility and Pathogenicity. Cell. 2021;184:64-75 e11.

[10] Yurkovetskiy L, Wang X, Pascal KE, Tomkins-Tinch C, Nyalile TP, Wang Y, et al. Structural and Functional Analysis of the D614G SARS-CoV-2 Spike Protein Variant. Cell. 2020;183:739-51 e8.

[11] Walls AC, Park YJ, Tortorici MA, Wall A, McGuire AT, Veesler D. Structure, function, and antigenicity of the SARS-CoV-2 spike glycoprotein. Cell. 2020;181:281-92.e6. 
[12] Letko M, Marzi A, Munster V. Functional assessment of cell entry and receptor usage for SARS-CoV-2 and other lineage B betacoronaviruses. Nat Microbiol. 2020;5:562-9.

356

357

358

359

360
[13] Wang Q, Zhang Y, Wu L, Niu S, Song C, Zhang Z, et al. Structural and Functional Basis of SARS-CoV-2 Entry by Using Human ACE2. Cell. 2020;181:894-904 e9.

[14] Shang J, Wan Y, Luo C, Ye G, Geng Q, Auerbach A, et al. Cell entry mechanisms of SARS-CoV-2. Proc Natl Acad Sci U S A. 2020;117:11727-34.

[15] Keech C, Albert G, Cho I, Robertson A, Reed P, Neal S, et al. Phase 1-2 Trial of a SARSCoV-2 Recombinant Spike Protein Nanoparticle Vaccine. N Engl J Med. 2020;383:2320-32.

[16] Walsh EE, Frenck RW, Jr., Falsey AR, Kitchin N, Absalon J, Gurtman A, et al. Safety and Immunogenicity of Two RNA-Based Covid-19 Vaccine Candidates. N Eng1 J Med. 2020;383:2439-50.

[17] Anderson EJ, Rouphael NG, Widge AT, Jackson LA, Roberts PC, Makhene M, et al. Safety and Immunogenicity of SARS-CoV-2 mRNA-1273 Vaccine in Older Adults. N Engl J Med. 2020;383:2427-38.

[18] McCallum M, De Marco A, Lempp FA, Tortorici MA, Pinto D, Walls AC, et al. N-terminal domain antigenic mapping reveals a site of vulnerability for SARS-CoV-2. Cell. 2021:S00928674(21)00356-1.

[19] Hoffmann M, Arora P, Gross R, Seidel A, Hornich BF, Hahn AS, et al. SARS-CoV-2 variants B.1.351 and P.1 escape from neutralizing antibodies. Cell. 2021:S0092-8674(21)003676.

[20] Tada T, Dcosta BM, Zhou H, Vaill A, Kazmierski W, Landau NR. Decreased neutralization of SARS-CoV-2 global variants by therapeutic anti-spike protein monoclonal antibodies. bioRxiv. 2021;2021.02.18.431897.

[21] Greaney AJ, Loes AN, Crawford KHD, Starr TN, Malone KD, Chu HY, et al. Comprehensive mapping of mutations in the SARS-CoV-2 receptor-binding domain that affect recognition by polyclonal human plasma antibodies. Cell Host Microbe. 2021;29:463-76 e6.

[22] Wang P, Nair MS, Liu L, Iketani S, Luo Y, Guo Y, et al. Antibody resistance of SARSCoV-2 variants B.1.351 and B.1.1.7. Nature. 2021. 
[23] Zhou H, Dcosta BM, Samanovic MI, Mulligan MJ, Landau NR, Tada T. B.1.526 SARS-

[24] Tada T, Dcosta BM, Samanovic MI, Herati RS, Cornelius A, Zhou H, et al. Convalescentefficacy of the ChAdOx1 nCoV-19 vaccine (AZD1222) against SARS-CoV-2: an interim analysis of four randomised controlled trials in Brazil, South Africa, and the UK. Lancet. 2021;397:99-111.

[28] Emary KRW, Golubchik T, Aley PK, Ariani CV, Angus B, Bibi S, et al. Efficacy of 398 ChAdOx1 nCoV-19 (AZD1222) vaccine against SARS-CoV-2 variant of concern 202012/01 399 (B.1.1.7): an exploratory analysis of a randomised controlled trial. Lancet. 2021;397:1351-62. Efficacy of Single-Dose Ad26.COV2.S Vaccine against Covid-19. N Engl J Med. 2021;384:2187-201. breakthrough rates of SARS-CoV-2 variants of concern in BNT162b2-mRNA-vaccinated 407 individuals. Nat Med. 2021;27:1379-84. 
410 [33] Choi A, Koch M, Wu K, Dixon G, Oestreicher J, Legault H, et al. Serum Neutralizing 411 Activity of mRNA-1273 Against SARS-CoV-2 Variants. J Virol. 2021:JVI0131321.

412 [34] Corbett KS, Werner AP, Connell SO, Gagne M, Lai L, Moliva JI, et al. mRNA-1273 413 protects against SARS-CoV-2 beta infection in nonhuman primates. Nat Immunol. 2021.

414

415

416

417

418

419

420

421

422

423

424

425

426

427

428

429

430

431

432

433

434

435

436

437

438
[35] Nelson J, Sorensen EW, Mintri S, Rabideau AE, Zheng W, Besin G, et al. Impact of mRNA chemistry and manufacturing process on innate immune activation. Sci Adv. 2020;6:eaaz6893.

[36] Hassett KJ, Benenato KE, Jacquinet E, Lee A, Woods A, Yuzhakov O, et al. Optimization of Lipid Nanoparticles for Intramuscular Administration of mRNA Vaccines. Mol Ther Nucleic Acids. 2019;15:1-11.

[37] Bahl K, Senn JJ, Yuzhakov O, Bulychev A, Brito LA, Hassett KJ, et al. Preclinical and Clinical Demonstration of Immunogenicity by mRNA Vaccines against H10N8 and H7N9 Influenza Viruses. Mol Ther. 2017;25:1316-27.

[38] John S, Yuzhakov O, Woods A, Deterling J, Hassett K, Shaw CA, et al. Multi-antigenic human cytomegalovirus mRNA vaccines that elicit potent humoral and cell-mediated immunity. Vaccine. 2018;36:1689-99.

[39] Vogel AB, Lambert L, Kinnear E, Busse D, Erbar S, Reuter KC, et al. Self-Amplifying RNA Vaccines Give Equivalent Protection against Influenza to mRNA Vaccines but at Much Lower Doses. Mol Ther. 2018;26:446-55.

[40] Whitt MA. Generation of VSV pseudotypes using recombinant DeltaG-VSV for studies on virus entry, identification of entry inhibitors, and immune responses to vaccines. J Virol Methods. 2010;169:365-74.

[41] Corbett KS, Edwards DK, Leist SR, Abiona OM, Boyoglu-Barnum S, Gillespie RA, et al. SARS-CoV-2 mRNA vaccine design enabled by prototype pathogen preparedness. Nature. 2020;586:567-71.

[42] Choi A, Koch M, Wu K, Chu L, Ma L, Hill A, et al. Safety and immunogenicity of SARSCoV-2 variant mRNA vaccine boosters in healthy adults: an interim analysis. Nat Med. 2021.

[43] Francica JR, Zak DE, Linde C, Siena E, Johnson C, Juraska M, et al. Innate transcriptional effects by adjuvants on the magnitude, quality, and durability of HIV envelope responses in NHPs. Blood Adv. 2017;1:2329-42. 
bioRxiv preprint doi: https://doi.org/10.1101/2021.04.13.439482; this version posted October 7, 2021. The copyright holder for this preprint (which was not certified by peer review) is the author/funder. All rights reserved. No reuse allowed without permission.

439 [44] Lan J, Ge J, Yu J, Shan S, Zhou H, Fan S, et al. Structure of the SARS-CoV-2 spike

440 receptor-binding domain bound to the ACE2 receptor. Nature. 2020;581:215-20.

441 
442 Tables

443 Table 1. S-protein substitutions in SARS-CoV-2 variants evaluated in this study.

\begin{tabular}{cc}
\hline Variant Name & Amino Acid Changes in S Protein Relative to Wuhan-Hu-1 \\
\hline D614G & D614G \\
\hline B.1.351 (Beta) & L18F, D80A, D215G, $4242-244$, R246I, K417N, E484K, \\
$(501 Y . V 2)$ & N501Y, D614G, A701V \\
\hline P.1 (Gamma) & L18F, T20N, P26S, D138Y, R190S, K417T, E484K, N501Y, \\
(501Y.V3) & D614G, H655Y, T1027I, V1176F \\
\hline B.1.427/B.1.429 (Epsilon) & S13I, W152C, L452R, D614G \\
(452R.V1, CAL.20C) & \\
\hline
\end{tabular}




\section{Figure Legends}

447 Fig. 1. Model of S protein. mRNA-1273.351 encodes the B.1.351 lineage S variant. Surface

448 representation of the trimeric S protein in the vertical view with the locations of surface-exposed

449 mutated residues highlighted in red spheres and labelled on the gray monomer. The inset shows

450 superimposition of ACE2 receptor domain and the RBD. S protein structure, 6VSB [3]. ACE2-

451 RBD structure, 6M0J [44].

452 ACE2 $=$ angiotensin converting enzyme 2; NTD = N-terminal domain; $\mathrm{RBD}=$ receptor binding 453 domain.

Fig. 2. S protein-binding antibody and neutralization of variant SARS-CoV-2 pseudoviruses

by serum from vaccinated BALB/c mice. a, BALB/c mice were immunized on a two-dose schedule with 1 or $10 \mu \mathrm{g}$ mRNA-1273, mRNA-1273.351, mRNA-1273.211 (1:1 mix of mRNAneutralization titers. Fold difference in neutralization against each virus was shown in text above 
469 is listed as text above each plot. The horizontal dotted line indicates the lower limit of quantitation

470 for NAb titer at $40 \mathrm{ID}_{50}$.

471 ELISA $=$ enzyme-linked immunosorbent assay; GMT = geometric mean titer; ID $_{50}=$ inhibitory

472 dilution factor; $\operatorname{IgG}=$ immunoglobulin $\mathrm{G}$; $\mathrm{Nab}=$ neutralizing antibody; ns, not significant; PBS =

473 phosphate-buffered saline; PsVN = pseudovirus neutralization titer

474

475

Fig. 3. S-protein binding antibody and neutralization of D614G and B.1.351 SARS-CoV-2

476

pseudoviruses by serum from $1 \mu \mathrm{g}$ mRNA-1273.351 boosted BALB/c mice. a, BALB/c mice

477 were immunized with 1 or $0.1 \mu \mathrm{g}$ mRNA-1273 (dose 1 on day 1 ; dose 2 on day 22) and were

478 boosted with 1 or $0.1 \mu \mathrm{g}$ mRNA-1273.351 on day 213 . b, Results from individual mouse sera (n

$479=5$ per group) are represented as dots on each figure, and the line is the mean of each group. The 480 horizontal dotted line indicates the lower limit of quantitation for $\log _{10}$ IgG titer at 1.398 . c, 481 BALB/c mice previously immunized with mRNA-1273 were given a third dose with $1 \mu \mathrm{g}$ mRNA482 1273.351, with PsVN assessed against wild-type D614G and B.1.351 prior to dose 3 (Day 212) 483 and 3 weeks after dose 3 (Day 233). Postdose 2 peak neutralization titer (Day 36) was referenced 484 against D614G. d, Fold rise in neutralization against both viruses from the boosting dose of 485 mRNA-1273.351. e, Fold difference in neutralization prior to and after dose 3. Postdose 2 peak 486 neutralization titer reference (D614G assay). The box indicates the GMT, which is listed as text 487 above each plot. The horizontal dotted line indicates the lower limit of quantitation for NAb titer 488 at $40 \mathrm{ID}_{50}$. Results from individual mouse sera is represented as dots on each figure, with lines 489 connecting the D614G and B.1.351 neutralization titers. 
bioRxiv preprint doi: https://doi.org/10.1101/2021.04.13.439482; this version posted October 7, 2021. The copyright holder for this preprint (which was not certified by peer review) is the author/funder. All rights reserved. No reuse allowed without permission.

490 ELISA = enzyme-linked immunosorbent assay; IgG = immunoglobulin G; PBS = phosphate-

491 buffered saline; PsVN = pseudovirus neutralization; GMT = geometric mean titer; ID $_{50}=$ inhibitory

492 dilution factor; $\mathrm{NAb}=$ neutralizing antibody. 


\section{$493 \quad$ Figures}

\section{$494 \quad$ Figure 1}

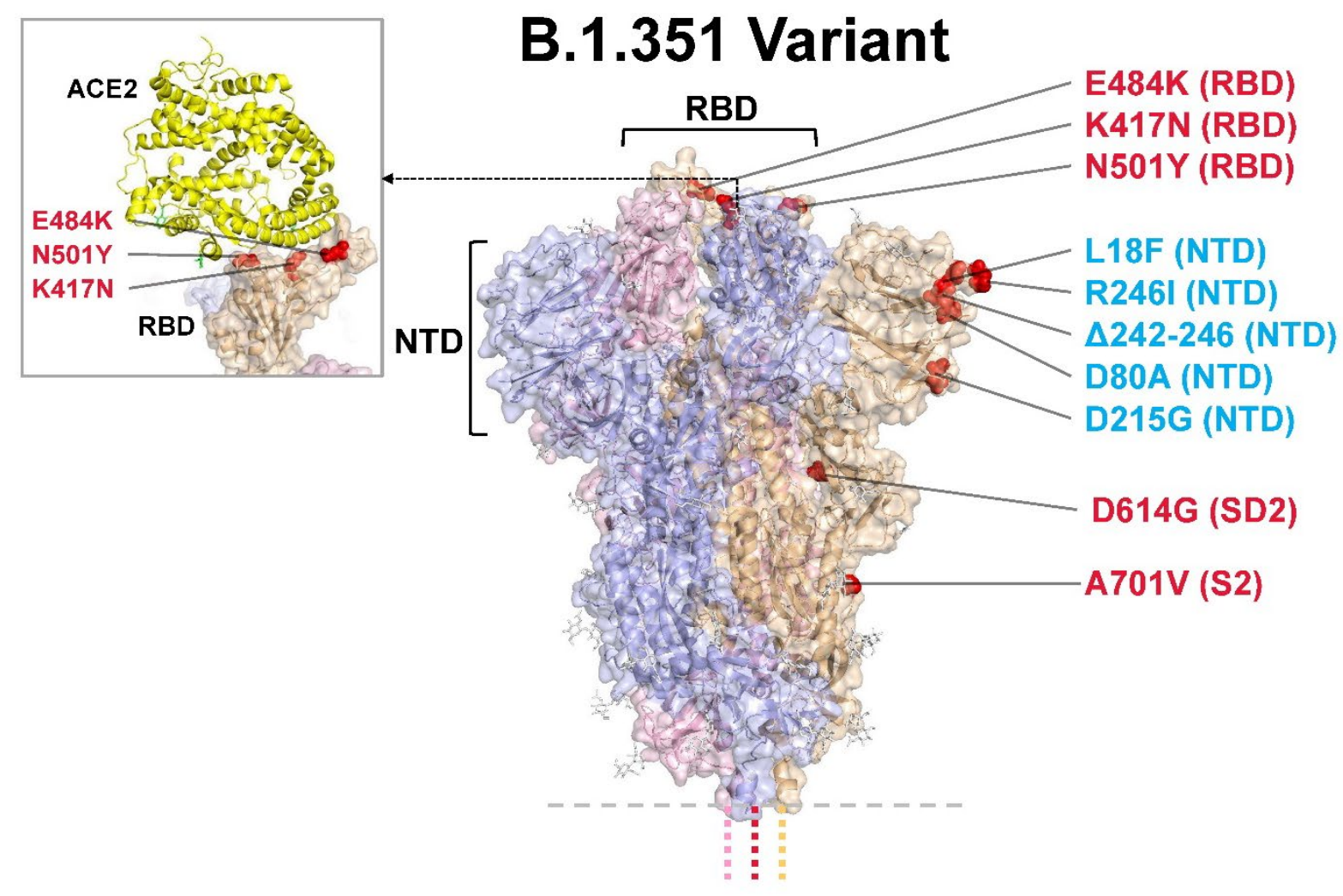


A

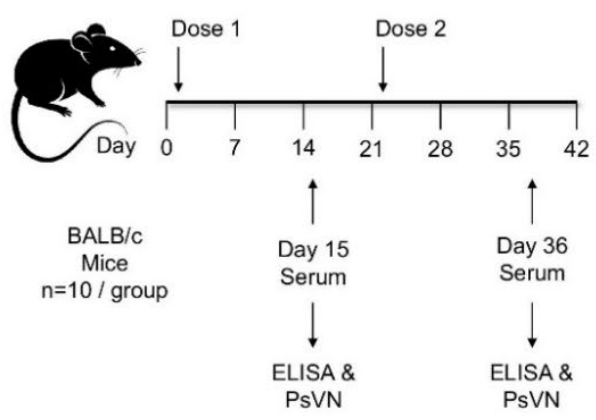

C

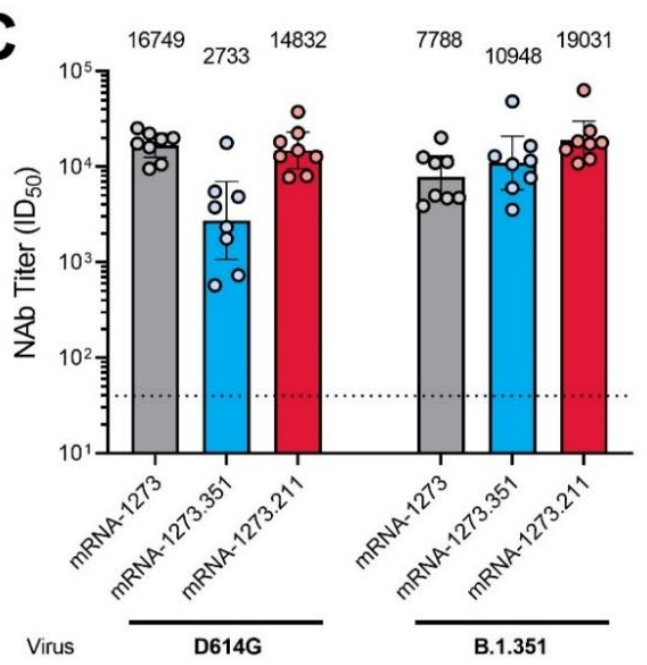

B
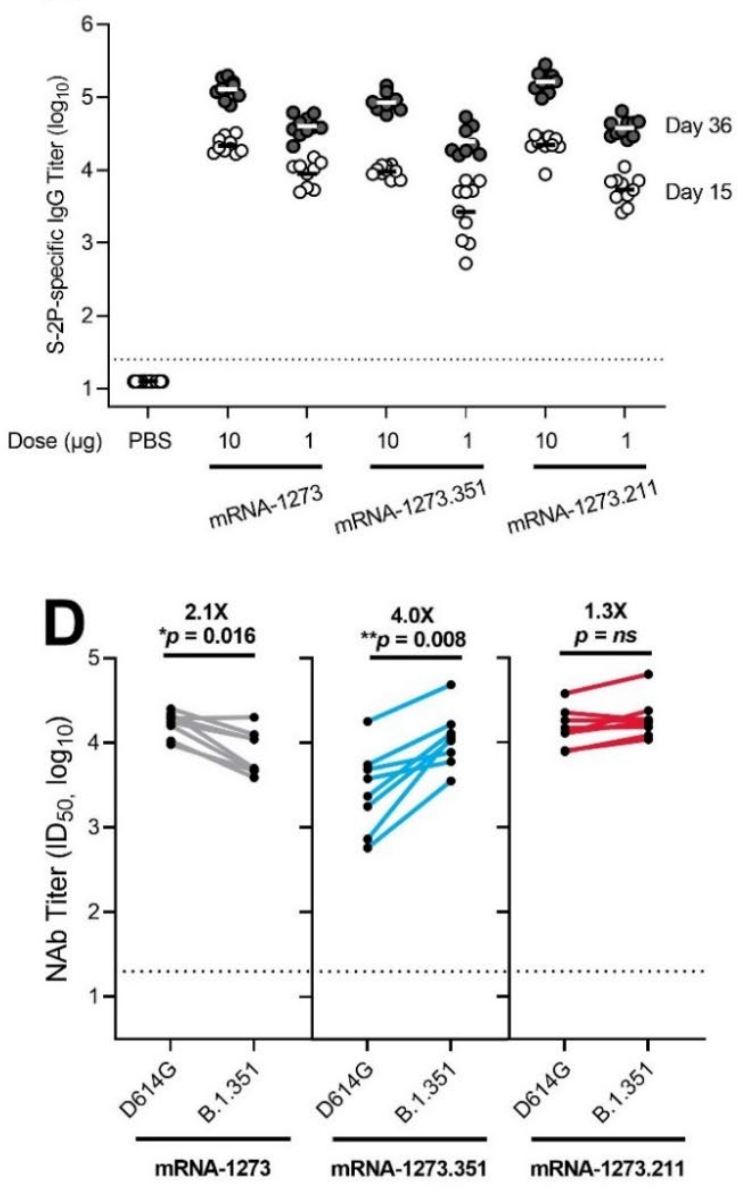

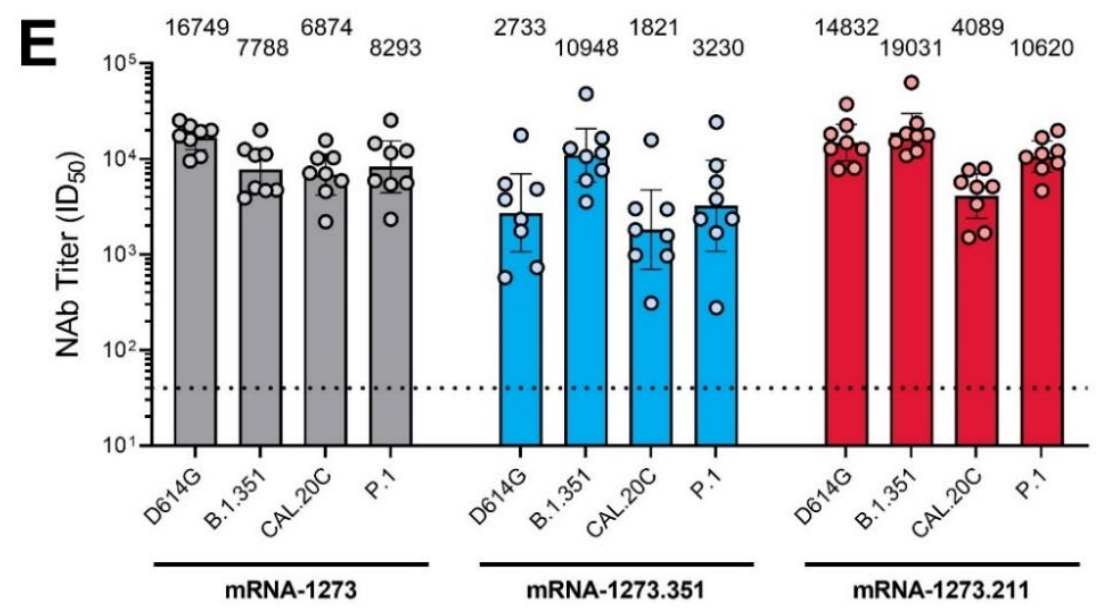




\section{Figure 3}

A

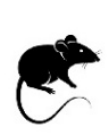

BALB/C Mice
$n=5 /$ group

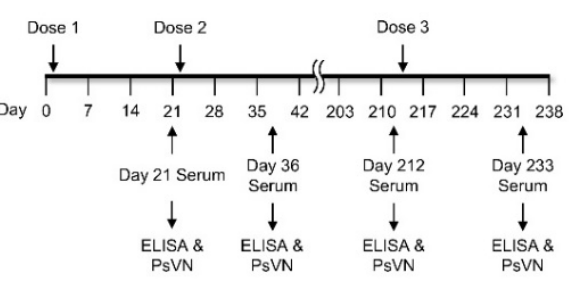

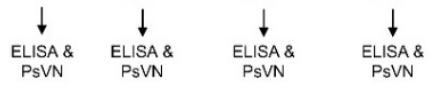

B

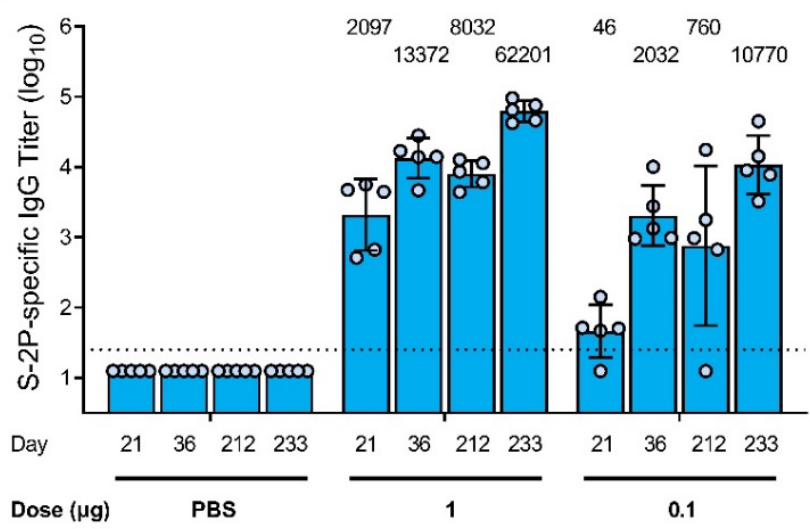

C

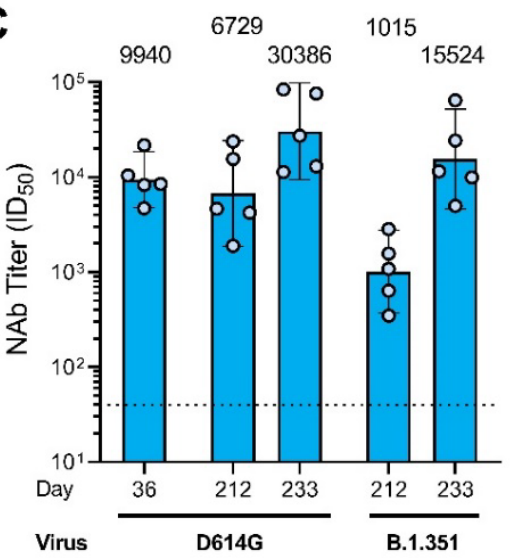

D

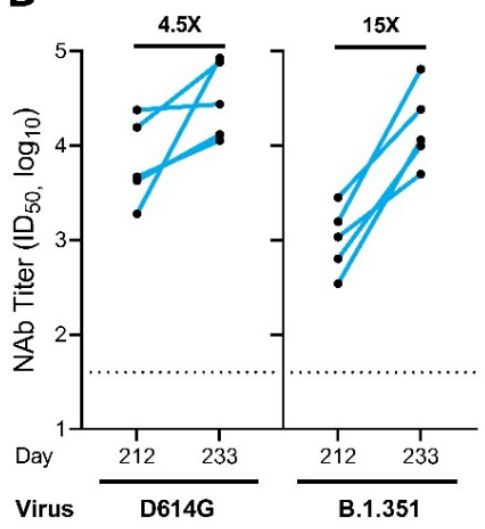

E

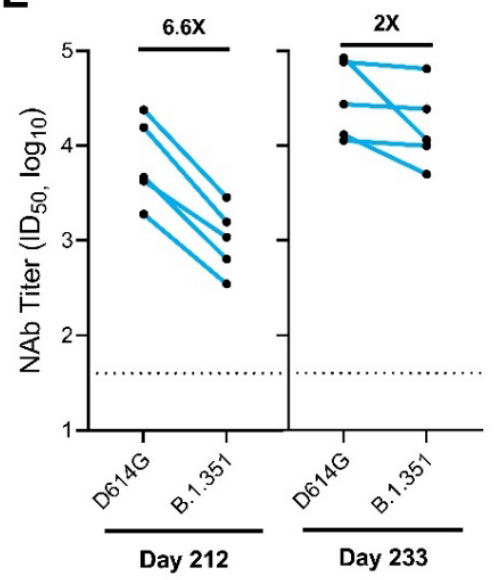

